\title{
Multi-trajectories automatic planner for StereoElectroEncephaloGraphy (SEEG)
}

\author{
E. De Momi - C. Caborni - F. Cardinale - G. Casaceli • \\ L. Castana - M. Cossu - R. Mai - F. Gozzo - S. Francione • \\ L. Tassi - G. Lo Russo - L. Antiga - G. Ferrigno
}

Received: 28 November 2013 / Accepted: 2 April 2014 / Published online: 20 April 2014

(C) CARS 2014

\begin{abstract}
Purpose StereoElectroEncephaloGraphy (SEEG) is done to identify the epileptogenic zone of the brain using several multi-lead electrodes whose positions in the brain are preoperatively defined. Intracranial hemorrhages due to disruption of blood vessels can cause major complications of this procedure $(<1 \%)$. In order to increase the intervention safety, we developed and tested planning tools to assist neurosurgeons in choosing the best trajectory configuration.

Methods An automated planning method was developed that maximizes the distance of the electrode from the vessels and avoids the sulci as entry points. The angle of the guiding screws is optimized to reduce positioning error. The planner was quantitatively and qualitatively compared with manually computed trajectories on 26 electrodes planned for three patients undergoing SEEG by four neurosurgeons. Quantitative comparison was performed computing for each trajectory using (a) the Euclidean distance from the closest vessel and (b) the incidence angle.

Results Quantitative evaluation shows that automatic planned trajectories are safer in terms of distance from the closest vessel with respect to manually planned trajectories. Qualitative evaluation performed by four neurosurgeons showed that the automatically computed trajectories would have been pre-
\end{abstract}

E. De Momi $(\bowtie) \cdot$ C. Caborni · G. Ferrigno

Department of Electronics, Information and Bioengineering

(DEIB), Politecnico di Milano, Milan, Italy

e-mail: elena.demomi@polimi.it

F. Cardinale - G. Casaceli $\cdot$ L. Castana $\cdot$ M. Cossu $\cdot$ R. Mai

F. Gozzo · S. Francione · L. Tassi · G. Lo Russo

"Claudio Munari" Centre for Epilepsy and Parkinson Surgery

Ospedale Niguarda Ca' Granda, Milan, Italy

L. Antiga

Orobix Srl, Bergamo, Italy ferred to manually computed ones in $30 \%$ of the cases and were judged good or acceptable in about $86 \%$ of the cases. A significant reduction in time required for planning was observed with the automated system (approximately 1/10). Conclusion The automatic SEEG electrode planner satisfied the essential clinical requirements, by providing safe trajectories in an efficient timeframe.

Keywords Computer-assisted surgery · Epilepsy · StereoElectroEncephaloGraphy

\section{Introduction}

The surgical treatment of drug-resistant epilepsy is mainly aimed at the removal or the disconnection of the Epileptogenic Zone (EZ), i.e., the "site of the emergence of the epileptic seizures and of their primary organization" [19], which is obtained through noninvasive pre-surgical investigation in most cases [13,20,32]. Nonetheless, 5-50\% of the subjects undergo intracranial electroencephalography to record both interictal and ictal brain activity $[17,31,35,36]$. Intracerebral electrodes allow the accurate sampling of lateral, mesial and inferior aspects of the cortical surface, of the white matter and of the subcortical gray matter structures, with a low complication rate [6].

In Stereo-Electro-Encephalo-Graphy (SEEG), a tailored, individualized arrangement of several intracerebral electrodes, covering the brain areas presumably involved by the discharge, allows for accurately defining the spatial and chronological organization of the EZ [23,24].

The correct positioning of intracerebral electrodes must address two essential requirements:

(i) accurate targeting of desired intracerebral structures;

(ii) minimizing the risk of complications such as intracranial bleeding, infections and cerebrospinal fluid leakage. 
Accurate trajectory planning is therefore demanded for an optimal implantation. The planning of the stereotactic trajectories is traditionally performed by a neurosurgeon, following the results of a multidisciplinary meeting with the epileptologists. The surgeon manually selects the entry points (EP) and the target points (TP) of every trajectory on dedicated software by visually inspecting multi-planar reconstructions (MPR) and 3D renderings of multimodal images $[7,8]$. Due to the large number of electrodes (up to 21 electrodes per patient in the Niguarda Hospital experience), SEEG planning is a hard and time-consuming procedure. Automatizing trajectory planning would therefore decrease planning time, preserving or even improving the safety.

Some authors proposed automatic needle trajectories planning methods for various kinds of surgeries. First attempts to automatize the planning process were aimed at planning safe trajectories for thermal radiofrequency ablations of hepatic tumors. Altrogge et al. [1] used a temperaturebased cost function for computing the best trajectories, maximizing the temperature of the tumor and minimizing the temperature of healthy tissue, avoiding the contact with the surrounding vessels. Baegert et al. [2,3] estimated best trajectories penalizing the ones that crossed vital organs in the abdomen, while Seitel et al. [26] introduced other optimization parameters such as instrument shape and penetration angle. Afterward, they focused their work on the brain district, where similar optimization criteria have to be accounted for in case of deep brain stimulation (DBS) procedures. Cost values are assigned to crossed voxels according to their distance to important brain structures [14]. Shamir et al. [27,28] proposed to automatically compute trajectories considering either the sum of each crossed voxel costs and the maximum crossed voxel cost, but did not provide any method for aggregating the two costs. Planner performances were retrospectively evaluated on eight patients in terms of quantitative parameters. Quantitative evaluations performed on optimization parameters can bias the presented results, since manual trajectories can consider other parameters, not taken into account in the planner. Qualitative trajectory judgments are therefore a more comprehensive analysis of the planner performances that cannot be disregarded when designing an automatic method. In [4], the vessels and the ventricles represent the structures that have to be avoided, and the best trajectory has to avoid sulci and crossing the midline too. The final trajectory score is computed combining the maximum risk and the sum of all the risks parameters for all surgical constraints using a weighted cost function. Quantitative results and preliminary qualitative results are reported. The method proposed by Liu et al. [22] for DBS trajectory planning (considering avoiding delicate brain structures) was qualitatively evaluated by two neurosurgeons on 20 trajecto- ries: automatically computed trajectories were preferred in $25 \%$ of the cases.

In [11], we proposed an automatic electrode trajectory planner for SEEG procedures: given an entry and a target region, the planner minimizes the value of a cost function optimizing some parameters such as distance from vessels and incidence angle. Each trajectory is computed depending only on the previous ones; thus, this planner will be called single planner (SP, hereafter). The aim of the present study is to introduce the multi-planner (MP) concept, which accounts for the spatial relationships among all the multiple trajectories, independently on the insertion order, i.e., the best trajectories configuration is computed in a single optimization run, thus working on more degrees of freedom at once and to qualitatively evaluate both the automatic methods against manual traditional planning.

\section{Materials and methods}

\section{The SEEG workflow at Niguarda Hospital}

The current Niguarda Hospital workflow for SEEG was elsewhere detailed by Cardinale et al. [6,8]. In summary, for every patient, 3D MR and brain 3D Digital Subtraction Angiography (3D-DSA) are preoperatively acquired without any frame or markers. 3D-DSA is obtained with a mobile cone-beam CT device (O-arm 1000 System, Medtronic Inc., Minneapolis, Minnesota, US, matrix $512 \times 512 \times 192$, voxel $0.4 \mathrm{~mm} \times 0.4 \mathrm{~mm} \times 0.8 \mathrm{~mm})$. A baseline dataset and additional datasets during the selective injection of iodinate contrast medium into the arteries are acquired. The baseline dataset is registered (6 Degrees of Freedom (DoFs); similarity function: correlation ratio [25]) to the contrast-enhanced one and subsequently subtracted from it, in order to obtain a skull masked dataset with the vessel tree. MR images are acquired using a 1.5T scanner (Intera Achieva, Philips Medical System, The Netherlands) and registered to the reference space (6 DoFs; similarity function: mutual information [33]). The study includes T1 3D FFE sagittal images, $0.90 \mathrm{~mm} \times 0.45 \mathrm{~mm} \times 0.45 \mathrm{~mm}$ voxel dimensions, without any inter-slice gap, reconstructed and reformatted on the axial plane with $560 \times 560 \times 220$ matrix, $0.45 \mathrm{~mm} \times$ $0.45 \mathrm{~mm} \times 0.9 \mathrm{~mm}$ voxel dimensions. The supratentorial brain tissue is segmented, and the hemispheres are split. The desired trajectories are preliminary defined with entry points (EPs) and target points (TPs) "roughly" positioned on this multimodal scenes at the time of the multidisciplinary staff meeting.

The co-registered datasets are loaded in Voxim (IVS Technology GmbH, Chemnitz, Germany), a stereotactic planning software providing dedicated tools for manually planning the needed trajectories. The surgeon manually selects the accu- 
rate and definitive EP and the TP of each trajectory by visually inspecting Multi-Planar Reconstructions (MPR), "surgeon's eye views" and 3D renderings of multimodal images. The day of the surgery an intraoperative preliminary cone-beam $\mathrm{CT}$ is performed, and the patient is registered to the stereotactic space. Pre-operative and intraoperative CT datasets are registered (6 DoFs, correlation ratio [25]), and the preplanned trajectories are moved into the surgical space. The electrodes (Microdeep ${ }^{\circledR}$, Dixi Medical, Besançon, France or Depth Electrodes Range 2069R, Alcis, Besançon, France) are implanted with the aid of the Neuromate ${ }^{\circledR}$ (Renishaw mayfield, Nyon, Switzerland). This stereotactic image- guided robotic system is driven by Voxim and automatically aligns the tool holder along the planned trajectories.

Linear registrations are performed with FSL [30] using FLIRT [18]. Freesurfer is used for brain segmentation and hemisphere splitting [9,12]. 3D Slicer is used to create the multimodal scenes [16]. The local Ethical Committee has approved the present study. All patients (or their guardians) sign an informed consent.

SP: the single-trajectory automatic planner

The single-trajectory automatic planner was described in [11]. The SP was developed for and was run in 3D Slicer 4.1 on an Apple iMac computer (Intel Quad-Core i7 3.1 GHz, 8 GB RAM).

Users are requested to input several parameters: allowed entry and target areas around the EPs and TPs rough fiducials, allowed minimum distance between the electrode and the closest vessel $\left(\theta_{v}\right)$, the minimum allowed distance between two electrodes $\left(\theta_{d}\right)$, the threshold angle $\left(\theta_{a}\right)$ between the electrode trajectory and the local vector perpendicular to the skull surface (i.e., the incidence angle $\alpha$ ), the maximum distance from the pre-planned EP and TP $\left(\theta_{e}\right.$ and $\left.\theta_{t}\right)$ and the maximum allowed cortex curvature value $\left(\theta_{S}\right)$ [29] at the EP. The number of possible entry points and target points depend on the image resolution and on the automatic segmentation described in section "The SEEG workflow at Niguarda Hospital." The user can also specify anatomical areas to be investigated and anatomical areas to be dodged.

A weight-based criterion was adopted to determine the best insertion trajectory, which maximizes, under suitable hard constraints, the following cost function $\mathrm{F}$ [5], which is the weighted sum of the distance from the vessel tree $\left(f_{v}\right)$ and of the insertion angle $\left(f_{a}\right)$ for each possible trajectory $t r_{i}$ :

$F\left(t r_{i}\right)=w_{v} \cdot f_{v}\left(t r_{i}\right)+w_{a} \cdot f_{a}\left(t r_{i}\right)$

where $w_{v}$ and $w_{a}$ are the weighting factors.

The cost function which expresses the distance from the vessel tree is the following: $f_{v}\left(t r_{i}\right)= \begin{cases}\frac{d_{\min }\left(t r_{i}\right)-d_{\min }}{d_{\max }-d_{\min },} & d_{\min }\left(t r_{i}\right)>\vartheta_{v} \\ \text { 'Discarded' } & d_{\min }\left(t r_{i}\right) \leq v_{v}\end{cases}$

where $d_{\min }\left(t r_{i}\right)$ is the minimum distance of the $i$ th shaft from the vessel tree and

$\left\{\begin{array}{l}d_{\max }=\max _{i} d_{\max }\left(t r_{i}\right) \\ d_{\min }=\min _{i} d_{\min }\left(t r_{i}\right)\end{array}\right.$

are the maximum and minimum distances of any point along any trajectory from the vessel tree, respectively.

The cost function which expresses the incidence angle $(\alpha)$ between the electrode shaft and the skull surface is defined as follows:

$f_{a}\left(t r_{i}\right)= \begin{cases}\frac{\alpha_{\max }-\alpha_{\max }\left(t r_{i}\right)}{\alpha_{\max }-\alpha_{\min }} & \alpha_{\max }\left(t r_{i}\right)<\vartheta_{a} \\ \text { 'Discarded' } & d_{\min }\left(t r_{i}\right) \geq \vartheta_{a}\end{cases}$

where $\alpha_{\max }\left(t r_{i}\right)$ is the angle between the $i$ th trajectory and the vector normal to the skull bone and:

$\left\{\begin{array}{l}\alpha_{\max }=\max _{i} \alpha_{\max }\left(t r_{i}\right) \\ \alpha_{\min }=\min _{i} \alpha_{\min }\left(t r_{i}\right)\end{array}\right.$

are the maximum and the minimum incidence angles of any trajectory, respectively. Trajectories for which $f_{v}\left(t r_{i}\right)$ or $f_{a}\left(t r_{i}\right)$ are "discarded" are removed from the pool. Among all possible trajectories, those, which cross user-defined brain structures, are considered not acceptable and removed from the pool, as well as all trajectories that do not respect the constrained minimum mutual distance $\left(\theta_{d}\right)$. It must be noted that the distance check in the SP is a serial process, so the choice of the first electrode changes the overall result. The algorithm also discards trajectories $t r_{i}$ for which the cortex curvature at the EP is below a pre-defined threshold value $\left(\theta_{s}\right)$.

MP: multi-trajectories automatic planner

Differently from the SP method, in the multi-trajectories automatic planner:

1. allowed entry/target areas are automatically computed propagating a distance field starting from rough EPs and TPs;

2. the best trajectories configuration is computed in a single optimization run. In this way, spatial optimization can be achieved independently from the choice of the electrodes planning sequence, leading in the practical case to a richer pool of possible trajectories.

\section{Allowed entry/target areas computation}

Starting from the rough definition of the EPs and TPs, a distance field is computed on the cortex surface model (40). Dis- 
tance thresholds from the candidate EPs/TPs points $\left(\theta_{e} / \theta_{t}\right.$, respectively) are defined by user. Points, whose distance is less than the user-defined distance thresholds and whose cortex curvature value is inferior to $\theta_{s}$, are considered as entry/target areas. Entry/target areas triangulated surfaces are sub-divided in triangles and then randomly resampled using the method described by Vitter [34]. The computation is implemented using the VTK scientific library (Kitware, Clifton Park, NY, USA).

\section{Automatic trajectories computation}

As reported in [11], a distance map from the vessel tree data is computed by means of the Danielsson's distance map algorithm [10], implemented using the ITK scientific library.

For each entry/target areas pair $(i, i=1 \ldots N$, where $N$ is the total number of multi-lead electrodes that have to be implanted), the set of possible entry points is $\left(E_{i}\right)$ and the set of target points $\left(T_{i}\right)$ are defined. The sets sizes are $S E_{i}$ and $S T_{i}$, respectively. Each possible trajectory $t r_{i j k}$ connects each entry point of region $E_{i}\left(E P_{i j}, j=1 \ldots S E_{i}\right)$ with each target point of region $T_{i}\left(T P_{i k}, k=1 \ldots S T_{i}\right)$. The total number of trajectories $(R)$ is:

$R=\sum_{i=1}^{N} S E_{i} \cdot S T_{i}$

All possible $M$ sized $N$ sets of trajectories configurations $T R_{h}$ are determined $(h=1 \ldots M)$ with $M=\prod_{i=1}^{N} S E_{i} \cdot S T_{i}$

A weight-based criterion was adopted to determine the best insertion trajectory configuration, which maximizes, under suitable hard constraints, the following cost function $F$ [11], which is the weighted sum of the distance from the vessel tree $\left(f_{v}\right)$ and of the insertion angle $\left(f_{a}\right)$ for each possible trajectories configuration $T R_{h}$ :

$$
\max _{h} F\left(T R_{h}\right)
$$

where

$F\left(T R_{h}\right)=w_{v} \cdot f_{v}\left(T R_{h}\right)+w_{a} \cdot f_{a}\left(T R_{h}\right)$

Trajectories not meeting the constraints are removed from the pool as in the SP.

\section{Surgeon assessment and possible replanning with MP}

The best $\mathrm{N}$-trajectories configuration $\left(\overline{T R}_{h}\right)$ is proposed to the neurosurgeon, and in case he/ she is not completely satisfied, he/ she can choose a subset of electrodes trajectories to be accepted or not accepted. Given the accepted trajectories, another plan is performed optimizing the same cost function reported above (7), changing the constraints in order to meet the surgeon preferences.
Validation protocol

A validation study was performed on 26 trajectories planned for three patients (Table 1) who underwent SEEG investigation since no obvious lesions were detected on MR images.

The validation protocol is shown in Fig. 1. On the basis of the topographic investigation strategy developed by the multidisciplinary team, the epileptologists selected the candidate EPs and TPs on the 3D Slicer multimodal scenes. We decided that the pre-planning was done by the epileptologists because of their lack of surgical planning experience. Anatomical details of the preliminary planning are reported in Table 1.

Voxim software application was used for the Manual planning (M) performed by the neurosurgeons. The multimodal scenes with the candidate EPs/TPs were used by them only as a reminder of the epileptological strategy to be followed. Manual planning was performed as described in "SP: the single-trajectory automatic planner" section, independently from the automatic planners, and the electrodes were implanted as above described.

Some months later (at least three), the SP and the MP were both ran in 3D Slicer 4.1, starting from the original multimodal scenes with the EPs/TPs selected by the epileptologists. The threshold was chosen to obtain a dataset as similar as possible to the original angiographic volume. The two weighting factors, $w_{v}$ and $w_{a}$, were set to 0.8 and 0.2 , respectively. The threshold distance from the closest vessel $\left(\theta_{v}\right)$ was set to $1.6 \mathrm{~mm}$ for the first $25 \mathrm{~mm}$ of electrode insertion (close to the entry area) and to $1 \mathrm{~mm}$ for the rest of the electrode length (which we afterward refer when talking about "depth vessel avoidance") since vessel avoidance is extremely important in the first millimeters at the cortical entry point [11]. The maximum angle between the vector normal to the skull surface and the electrode trajectory $\left(\theta_{a}\right)$ was set to $40^{\circ}$, the minimum distance between two electrodes $\left(\theta_{d}\right)$ was set to $3.3 \mathrm{~mm}$ and the cortex curvature threshold value $\left(\theta_{s}\right)$ was set to 0.15 . All those parameters were defined in [5] (Table 2).

\section{Quantitative evaluation}

The manually planned trajectories were exported from Voxim and imported into 3D Slicer. Quantitative comparison was performed by computing for each trajectory (a) the Euclidean distance from the closest vessel along the first $25 \mathrm{~mm}$ of insertion and (b) the incidence angle (guiding screws angle). $\mathrm{M}, \mathrm{SP}$ and MP results were compared using nonparametric Friedman test with Bonferroni correction.

\section{Qualitative evaluation}

The automatically computed trajectories were exported from 3D Slicer and imported into Voxim. All the trajectories (from 
Table 1 Patients data and exploration characteristics

\begin{tabular}{|c|c|c|c|c|c|c|c|}
\hline Case \# & Age & Pattern & Trajectory & $\begin{array}{l}\text { Anatomical structures } \\
\text { to be investigated }\end{array}$ & $\begin{array}{l}\text { Anatomical structures } \\
\text { to be avoided }\end{array}$ & EP definition & TP definition \\
\hline \multirow[t]{7}{*}{1} & \multirow[t]{7}{*}{41} & \multirow[t]{7}{*}{ Frontal (Left) } & G & F3-pars triangularis, gyrus cinguli & \multirow[t]{7}{*}{$\begin{array}{l}\text { Lateral ventricles, } \\
\text { thalamus, cerebellum }\end{array}$} & \multirow[t]{7}{*}{ Cortex surface } & \multirow[t]{7}{*}{ MPR } \\
\hline & & & $\mathrm{O}$ & F3-pars orbitalis, gyrus rectus & & & \\
\hline & & & $\mathrm{E}$ & $\mathrm{F} 2$, gyrus frontalis medialis & & & \\
\hline & & & Q & $\mathrm{F} 2$, gyrus cinguli & & & \\
\hline & & & $X$ & $\mathrm{~F} 1$, gyrus rectus & & & \\
\hline & & & Y & $\mathrm{F} 1$, cortex orbitalis & & & \\
\hline & & & $\mathrm{Z}$ & F2. gyrus cinguli (genu) & & & \\
\hline \multirow[t]{10}{*}{2} & \multirow[t]{10}{*}{35} & \multirow[t]{10}{*}{ Frontal (Left) } & $\mathrm{N}$ & Gyrus precentralis, gyrus cinguli & \multirow[t]{10}{*}{$\begin{array}{l}\text { Lateral ventricles, } \\
\text { thalamus, cerebellum }\end{array}$} & \multirow[t]{10}{*}{ Cortex surface } & \multirow[t]{10}{*}{ MPR } \\
\hline & & & $\mathrm{H}$ & $\mathrm{F} 2$, gyrus cinguli & & & \\
\hline & & & G & F3-pars triangularis, gyrus cinguli & & & \\
\hline & & & $\mathrm{O}$ & F3-pars orbitalis, gyrus cinguli (genu) & & & \\
\hline & & & $\mathrm{X}$ & $\mathrm{F} 1$, gyrus frontalis medialis & & & \\
\hline & & & $\mathrm{Y}$ & F1, gyrus cinguli (genu) & & & \\
\hline & & & $\mathrm{Z}$ & F1, gyrus cinguli & & & \\
\hline & & & $\mathrm{K}$ & F1, gyrus cinguli & & & \\
\hline & & & $\mathrm{R}$ & Gyrus precentralis, gyrus cinguli & & & \\
\hline & & & $\mathrm{L}$ & F2, gyrus cinguli & & & \\
\hline \multirow[t]{9}{*}{3} & \multirow[t]{9}{*}{48} & \multirow[t]{9}{*}{$\begin{array}{l}\text { Temporo-insulo- } \\
\text { perisilvian } \\
\text { (Right) }\end{array}$} & $X$ & Gyrus precentralis, insula & \multirow[t]{9}{*}{$\begin{array}{l}\text { Lateral ventricles, } \\
\text { thalamus, cerebellum }\end{array}$} & \multirow[t]{9}{*}{ Cortex surface } & MPR \\
\hline & & & $\mathrm{R}$ & Gyrus postcentralis, insula & & & MPR \\
\hline & & & $\mathrm{P}$ & Lobus parietalis inferior, precuneous & & & Cortex surface \\
\hline & & & $\mathrm{N}$ & Gyrus postcentralis, lobus paracentralis & & & Cortex surface \\
\hline & & & M & Gyrus precentralis, gyrus cinguli & & & Cortex surface \\
\hline & & & I & $\mathrm{F} 2$, gyrus frontalis medialis & & & Cortex surface \\
\hline & & & $\mathrm{H}$ & Gyrus precentralis, gyrus cinguli & & & Cortex surface \\
\hline & & & $\mathrm{F}$ & $\mathrm{F} 2$, gyrus cinguli & & & Cortex surface \\
\hline & & & $\mathrm{E}$ & F3-pars triangularis, gyrus cinguli & & & Cortex surface \\
\hline
\end{tabular}

M, SP and MP methods) were randomly labeled and blindly presented to four neurosurgeons (FC, MC, GC and LC). They were asked to separately evaluate five parameters for each trajectory:

(a) depth vessel avoidance;

(b) vessel avoidance at the entry point;

(c) adherence to the epileptologist plan (is the proposed trajectory crossing the wanted structure?) at the entry point;

(d) adherence to the epileptologist plan at the target point and

(e) incidence angle.

Every property was ranked as "good," "acceptable" or "discarded" for each trajectory planned using M, SP and MP. Percentages of good, acceptable or discarded trajectories were analyzed grouping self-tests (i.e., the surgeon who performed the manual plan is blindly judging SP, MP and his/her manually planned trajectories) and out-tests (i.e., the neurosurgeon is judging trajectories manually planned by another neurosurgeon). Trajectories that have been preferred (among not discarded trajectories) were also counted.

Self-test and out-test, M, SP and MP results of preferred and discarded trajectories populations were compared using two-tailed Fisher exact test. $p<0.05$ was considered as evidence of findings not attributable to chance. The statistical analysis was performed with Matlab ${ }^{\circledR}$ v.R2010a.

Fleiss' kappa analysis was perform to assess the four surgeons agreement on the 26 trajectories classification in "good," "acceptable" or "discarded" for each considered method $(p<0.05)[15]$.

\section{Results}

In the following, we report the first evaluation results obtained the 26 trajectories coming from three different patients. As not all the rough entry points proposed by the 

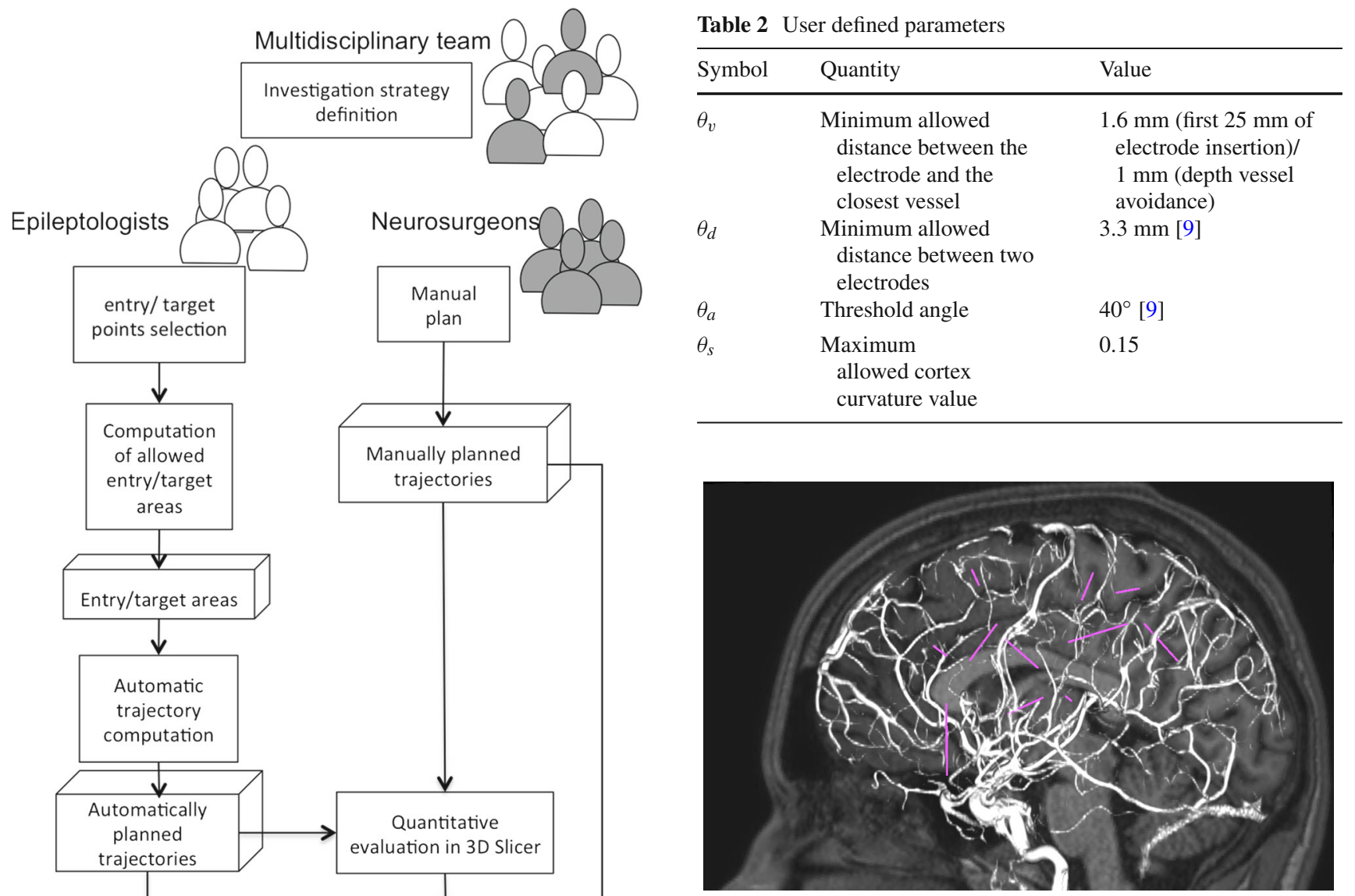

Fig. 2 Output of the automatic planner. Electrodes trajectories are displayed (in purple) in the 3D visualization environment, where medical images can be overlaid. The 3D environment can be interactively navigated

ment and on the 2D overlaid orthogonal views. The user can navigate and explore each trajectory using the surgeon eye projection.

Fig. 1 Validation protocol flowchart. The multidisciplinary team is composed by a pool of epileptologists and neurosurgeons, who took part in the methods evaluation, as detailed in the paper

epileptologists were considered feasible by the neurosurgeons, only a subset of trajectories for each patient was evaluated. This was due to the fact that the neurosurgeons experience suggested different, more accurate, exploration strategies. The total number of planned trajectories was 24 in case of SP and 26 for both the M and MP methods. In fact, the SP did not find any suitable solution for two pairs of entry/target areas, due to the highlighted limitation of dependence upon the planning order. The MP planning took approximately 10 min per patient.

Figure 2 shows the output of the automatic planned electrodes trajectories displayed in the 3D visualization environ-

\section{Quantitative evaluation}

The comparison between the manual planning and the two automatic planners is shown in Fig. 3, which displays (a) the Euclidean distance from the closest vessel along the first $25 \mathrm{~mm}$ of insertion and (b) the trajectory incidence angle for each patient and for each trajectory. Trajectories of all three patients were the observation units. Trajectories computed using the MP had a greater distance from vessels along the first $25 \mathrm{~mm}$ of electrode insertion with respect to the $M$ $(p=0.009)$. There is not any significant difference between the two automatic algorithms. Moreover, no statistically significant difference was computed considering the trajectory incidence angle. Nevertheless it should be kept into account that the SP was not able to suitably plan all the required trajectories (see Table 3, 24/26 and 72/78). 

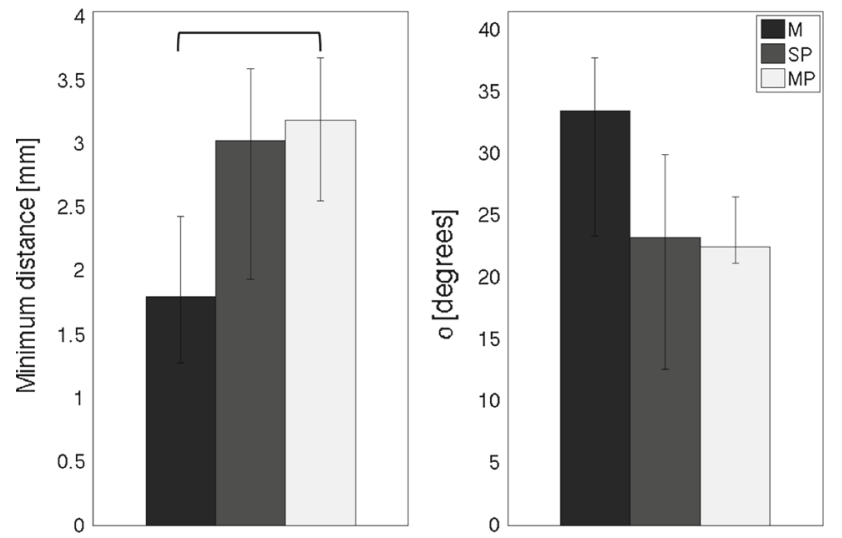

Fig. 3 Comparison between the manual planning $(\mathrm{M})$ and the two automatic planning methods (SP and MP) considering the minimum vessel distance and the insertion angle for 26 trajectories. Median values and inter-quartile range (25th and 75 th percentiles, vertical bars) are shown. Horizontal bars indicate statistical significant difference $(p<0.05)$

Table 3 Number of preferred and discarded trajectories for the manual planning (M) and the two automatic planning methods (SP and MP)

\begin{tabular}{lcll}
\hline Test & M & SP & MP \\
\hline \multicolumn{2}{l}{ Number of preferred trajectories } & & \\
Self-test & $7 / 26$ & $9 / 24$ & $10 / 26$ \\
Out-test & $30 / 78$ & $39 / 72$ & $22 / 78$ \\
Number of discarded trajectories & & \\
Self-test & $7 / 26$ & $2 / 24$ & $2 / 26$ \\
Out-test & $19 / 78$ & $8 / 72$ & $11 / 78$ \\
\hline
\end{tabular}

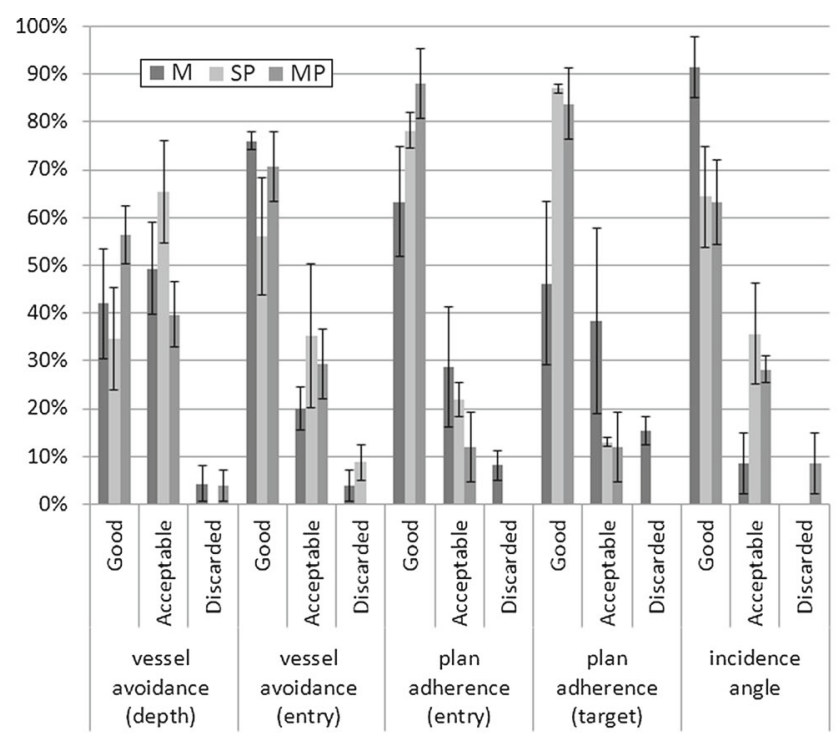

Fig. 4 Qualitative analysis results of the manual planning (M) and the two automatic planning methods (SP and MP) (a) depth vessel avoidance; (b) vessel avoidance at the entry point; (c) adherence to the epileptologist plan at the entry point; (d) adherence to the epileptologist plan at
Qualitative evaluation

Since the proportion of discarded trajectories and patient ID were found to be two independent classification criteria (two-tailed Fisher exact test: $p=0.27$ ), all trajectories were grouped together. Thus, the trajectories were the units of observation ( 26 trajectories).

The number of globally preferred trajectories and the number of discarded trajectories are reported in Table 3, considering self-test and out-test preferences. In general (Table 3), automatically computed trajectories were preferred in $40 \%$ of the cases during self-test and $30 \%$ during out-test. The SP discarded trajectories are only 8 out of $72(11.1 \%)$ tested trajectories, and the MP discarded trajectories are 11 out of $78(14.1 \%)$. No significant difference was found among the evaluation, whether it was self-test or out-test and among the evaluated method ( $p=0.38$ for preferred trajectories and $p=0.9$ for discarded trajectories, respectively).

Figure 4 by the four neurosurgeons, in terms of percentages of trajectories that were judged as good, acceptable or discarded. Figure 4, on the left, shows the mean percentage values, averaging for the 3 self-tests ( 3 neurosurgeons judging each one of the three patients) and, on the right, the mean percentage values, averaging for the nine out-tests (four neurosurgeons judging all the three patients, except the three selfplanned) considering the following evaluation parameters

(a) depth vessel avoidance;

(b) vessel avoidance at the entry point;

(c) adherence to the epileptologist plan at the entry point;

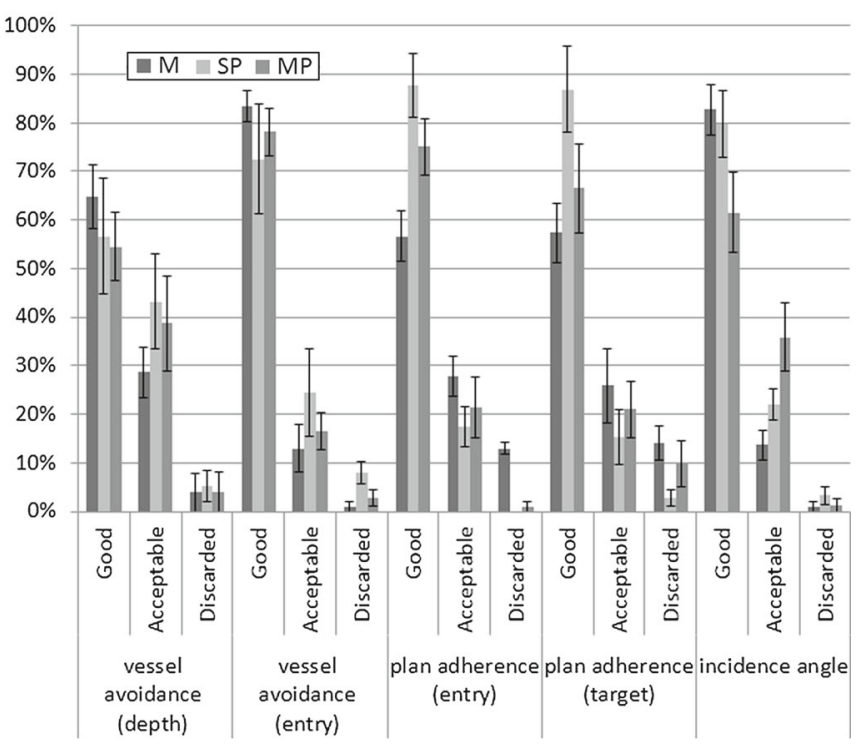

the target point and (e) incidence angle. On the left, self-tests results, on the right out-test results. Mean values are shown, vertical bars indicate the standard deviation" 
Table 4 Surgeons agreement indexes of the manual planning (M) and the two automatic planning methods (SP and MP) using Fleiss'kappa analysis

\begin{tabular}{llll}
\hline Test & M & SP & MP \\
\hline Evaluation parameter & & & \\
Vessel avoidance (depth) & Fair (0.29) & Fair (0.34) & Fair (0.30) \\
Vessel avoidance (entry) & Poor (-0.04) & Fair (0.26) & Fair (0.37) \\
Plan adherence (entry) & Moderate (0.52) & No & No \\
Plan adherence (target) & Fair (0.27) & Fair (0.21) & Slight $(0.08)$ \\
Incidence angle & Slight (0.16) & Fair (0.20) & Fair $(0.21)$ \\
\hline
\end{tabular}

(d) adherence to the epileptologist plan at the target point and

(e) incidence angle.

In case of self-test, each surgeon is in general confirming the manual choices $(75 \%$ of the cases considering the vessel avoidance at the entry region), and in about $70 \%$ of the cases, the automatically planned trajectories are also considered good. The same numbers are also confirmed during the out-test evaluation ( $80 \%$ approximately). The automatic plan is judged as a good plan considering vessel avoidance in deep brain regions, and that is particularly evident in case of self-test.

Fleiss' kappa analysis (Table 4) shows that, as far as the most important qualitative measure is concerned, i.e., vessel avoidance at the entry point, there is a poor agreement of the surgeons judging the manual method performances, despite it is the best in the number of "good" trajectories. Surgeons' agreement in judging the MP method, when the plan adherence is concerned, is inferior with respect to both the $\mathrm{M}$ and the SP methods.

\section{Discussion}

This study reports on a new automatic method for assisting neurosurgeons in SEEG planning, optimizing the entire batch of electrode trajectories on the basis of user-defined constraints and thus assisting the decision-making process. The new MP presented in this study is a software tool for automatic intracerebral trajectory planning dedicated to SEEG, optimizing all the trajectories considering their positions relative to each other. To the best of our knowledge, this is the first attempt of implementing such a feature, and this is the most obvious strength of this study. Moreover, we performed both quantitative and qualitative clinical validation of the MP, comparing its output with the SP (an older and simpler version of the automatic planner) and, most important, with the usual manual planning performed by three experienced neurosurgeons. The most important limit of this study is the small sample size, due to the hard time-consuming validation protocol, although the interesting sample is the one of the trajectories. The number of evaluated patients was kept low in order to have all the neurosurgeons evaluate the trajectories chosen by others, which is a time-consuming task, not performed in clinical practice. Such an evaluation gave us the possibility to understand the agreement ratio between neurosurgeons and with the planners. Nevertheless, the number of evaluated trajectories (i.e., 26) gives enough statistical power for the quantitative analysis (greater than $90 \%$ using a $T$ student test).

Another important limit is that the quality of 3D-DSA images visualization is slightly different for $\mathrm{M}$ and the automatic planners (SP and MP). As said, the M method used the Voxim environment, while the SP and MP were both run in 3D Slicer development environment.

For the qualitative comparison, there are a total of 26 manually planned trajectories. The planners were run once to compute 26 and 24 trajectories. Each surgeon was presented with a total $(26$ manual $+24 \mathrm{SP}+26 \mathrm{MP})$ trajectories to evaluate for a total of $(104$ manual $+96 \mathrm{SP}+104 \mathrm{MP})$ trajectories. Of the total counts, the self-test includes (26 manual + $24 \mathrm{SP}+26 \mathrm{MP}) 76$ trajectories and the out-tests include (78 manual + $72 \mathrm{SP}+78 \mathrm{MP}) 228$ trajectories. As far as the quantitative analysis is concerned, the two automatic methods (SP and MP) electrode trajectories obtained increased distance to the closest blood vessel at the EP with respect to manually planned trajectories of approximately $1.5 \mathrm{~mm}$, considering that the median localization error at the cortical entry point is reported to be $0.78 \mathrm{~mm}$ [7]. Both SP and MP performed equally well when finding a solution (SP was not always successful). The slight improvement in the vessel distance at the EP obtained using the MP method is likely due to the cost function optimization method, which optimizes the best trajectories configuration, rather than computing each trajectory separately and checking for collisions (which put importance on the order of planning). Nevertheless, the improvement of the MP over the SP is not significant, likely due to the relatively small sample size (i.e., 26 trajectories were analyzed). Cardinale et al. [7] reported on a multivariate analysis suggesting that the skull incidence angle, among other variables, significantly affects the localization error in SEEG implantations (the closer to the orthogonality, the smaller the error). In the present study, both MP and SP succeeded in optimizing the incidence angle, even if the angles were not significantly lower when compared to M. With respect to the SP, the MP allows for a spatial optimization, which results in finding possible solutions for all the planned trajectory configurations, as demonstrated by the achieved results since the SP was not able to find solutions for 2 trajectories.

Similarly to the present study, Shamir et al. $[27,28]$ retrospectively evaluated their results on the quantitative parameters in a series of eight patients that underwent DBS. Some other groups made also a qualitative evaluation of their automatic planner. Bériault et al. [4] evaluated the results of their 
automatic planner not only on the basis of the cost function minimization, but also considering qualitative criteria. Liu et al. [22] reported on the qualitative evaluation performed by two neurosurgeons on 20 DBS trajectories: automatically computed trajectories were preferred in $25 \%$ of the cases. Accordingly, we performed also a qualitative evaluation of our automatic planners.

In terms of clinical usability, the MP outperforms the SP since all the possible trajectory configurations are computed and saved in a priority list. If the surgeon is not satisfied with some trajectories, those can be excluded by the final set and another configuration can be almost immediately computed. Also, as reported in [11], automatic planning allows for a significant reduction in the planning time, where the MP halves the computational time with respect to the SP.

In general, automatic methods optimization criteria and weights are defined a priori by the end-users. In [27], the authors proved the inter-users validity of user-defined optimization weight criteria, proving the method valuable clinical assistance. Indeed, they performed the test on two users and 20 trajectories, and they found that differences of weights preferences between neurosurgeons were not significant. We proposed a qualitative evaluation aimed at verifying the consistency of each neurosurgeon manual plan, blindly compared with automatically computed trajectories (self-test) and also the agreement between different neurosurgeons, blindly comparing trajectories manually planned by other neurosurgeons with the ones automatically planned (outtest). Our analysis is therefore a comprehensive assessment of the best planning strategy. We did not find any inconsistency between the self-test and the out-test, independently on the neurosurgeon level of experience. This is likely due to the fact that all the neurosurgeons belong to the same hospital team.

Even if the planner optimized only three of the evaluated criteria (a)-(b)-(e), the neurosurgeons evaluated also the adherence to the epileptologist input (c)-(d). In general, automatically computed trajectories were preferred in $30-40 \%$ of the cases during self-test and during out-test, which is greater than what found in [22] for the specific DBS target.

Even if there is no unanimous acceptance of the $k$ values interpretation, the use of kappa statistics to assess examiner agreement for categorical outcomes has grown exponentially. Our analysis, based on the three classification criteria (good, acceptable and discarded) of the three proposed methods performed by four surgeons, suggested that there is a general agreement among the four experts, even if some disagreement appear when judging manual trajectories and the automatic methods adherence at the entry point. It has to be underlined that, even if the categories "good" and "acceptable" were considered separated, the qualitative judgment could be overlapping.

The automatic method thus proved to be an useful proposal for definitive neurosurgical SEEG planning. In case of automatic planning, the reasons for discarding computed trajectories were:

1. the amount of the crossed gray matter was too limited or

2. vessels, not visible from the automatic tree segmentation, were hit.

When neurosurgeons manually plan using the Voxim application, they use, in fact, the native angiography dataset, not the binarized one. Future implementation plans are directed toward the usage of maximum intensity projection technique (MIP) [21] that allows increasing the vessels tree visibility for the input dataset for the distance map creation.

Do these results mean that the automatic planners planned better than the neurosurgeons? The answer is yes, but limited to the parameters that were included in the algorithm. It has to be underlined that in SEEG procedures the target is not just the TP itself, but also the whole trajectory. SEEG differs, in fact, from DBS and biopsy procedures, where the interest is focused mostly on the deep target. The multi-lead electrodes record brain activity of white and gray matter along the whole trajectory that lies between EP and TP. The neurosurgeon could prefer a trajectory, which is closer to vessels when considering other optimization criteria, such as the maximization of the number of intracortical contacts. Depending on the structures to be explored (whether it is on the external or in the internal cortex), the weight of vessel distance should change: in case the neurosurgeon wants to maximize the number of recording contacts in the gray matter on the external cortex, he/ she could release the constraint that penalizes trajectories too close to vessels at the entry area. In some particular situations, such as in case of nodular heterotopia, the deep target region is small and well specified: In that case, the single planner strategy should be adopted for sampling the signals coming from the nodule and for thermocoagulating it after the multi-lead electrode implantation, while the multi-planner can be used for planning trajectories that have to cross extranodular structures. The first gives information on the seizure origin, the latter gives information on the seizure diffusion. Another possibility is to use in MP different optimization thresholds for the electrode that must hit the target.

In the future, more constraints will be included in the algorithm, and the end-user will be able to set more parameters. For example, we are going to develop a tool to set the proportion of gray/white matter to be explored. We will also implement a list of parcellated cortical areas [12] to be chosen on the basis of the electro-clinical context. Being modular in the required input information and based on open source libraries, the presented methodology could be easily extended to other clinical centers, performing SEEG planning. Moreover, further developments will be aimed at extending the planner concepts to other keyhole procedures, 
potentially performed using robots as alignment tools, such as drug delivery procedures.

\section{Conclusion}

Both the automatic planners proved to be clinically valuable for assisting SEEG planning and potentially other neurosurgical procedures such as stereotactic procedures for DBS and brain biopsies. The MP method outperformed the SP in terms of computed feasible trajectories since the potential entry areas were automatically computed considering the cortex curvature. Such methods are not replacing the manual neurosurgeon plan, rather can be used as possible suggestion of new safe trajectories, which take into account user-defined specific constraints. In fact, in our experience, some automatically computed trajectories were even preferred to manual ones.

In the time being, the time needed for performing the SEEG planning will be likely reduced implementing such an automatic planner in the routine workflow.

Acknowledgments This work was supported in part by the EU project ACTIVE FP7 ICT 270460, by Renishaw mayfield (Switzerland) and by Renishaw (UK).

Conflict of interest Author F.C. is a consultant to Renishaw-mayfield, the manufacturer of Neuromate robotic system, and a former consultant to Medtronic, the manufacturer of the O-arm.

\section{References}

1. Altrogge I, Kröger T, Preusser T, Büskens C, Pereira PL, Schmidt D, Weihusen A, Peitgen HO (2006) Towards optimization of probe placement for radio-frequency ablation. In: Larsen R, Nielsen M, Sporring J (eds) International conference on medical image computing and computer assisted intervention (MICCAI), LNCS 4190. Springer, Berlin, pp 486-493

2. Baegert C, Villard C, Schreck P, Soler L (2007) Precise determination of regions of interest for hepatic RFA planning. Stud Health Technol Inform 125:31-36

3. Baegert C, Villard C, Schreck P, Soler L, Gangi A (2007) Trajectory optimization for the planning of percutaneous radiofrequency ablation of hepatic tumors. Comput Aided Surg 12(2):82-90

4. Bériault S, Subaie FA, Collins DL, Sadikot AF, Pike GB (2012) A multi-modal approach to computer-assisted deep brain stimulation trajectory planning. Int J Comput Assist Radiol Surg 7(5):687-704

5. Caborni C, De Momi E, Antiga L, Hammoud A, Ferrigno G, Cardinale F (2012) Automatic trajectory planning in stereoelectroencephalography image guided neurosurgery. Int J Comput Assist Radiol Surg 7(1):S126-S128

6. Cardinale F, Cossu M, Castana L, Casaceli G, Schiariti M, Miserocchi A, Fuschillo D, Moscato A, Caborni C, Arnulfo G, Lo Russo G (2013) StereoElectroEncephaloGraphy: surgical methodology, safety and stereotactic application accuracy in five hundred procedures. Neurosurgery 72(3):353-366

7. Cardinale F, Cossu M, Castana L, Schiariti M, Miserocchi A, Casaceli G, Caborni C, Moscato A, Lo Russo G (2012) Five hundreds stereoelectroencephalography (SEEG) procedures for epilepsy surgery: a retrospective analysis of clinical safety and in vivo application accuracy. Int J Comput Assist Radiol Surg 7(1):S124-S125

8. Cardinale F, Miserocchi A, Moscato A, Cossu M, Castana L, Schiariti MP, Gozzo F, Pero G, Quilici L, Citterio A, Minella M, Torresin A, Lo Russo G (2012) Talairach methodology in the multimodal imaging and robotic era. In: Scarabin J (ed) Stereotaxy and epilepsy neurosurgery. John Libbey Eurotext, London, pp 245-272

9. Dale AM, Fischl B, Sereno MI (1999) Cortical surface-based analysis, I: segmentation and surface reconstruction. Neuroimage 9(2):179-194

10. Danielsson PE (1980) Euclidean Distance Mapping. Comput Graph Image Process 14:227-248

11. De Momi E, Caborni C, Cardinale F, Castana L, Casaceli G, Cossu M, Antiga L, Ferrigno G (2013) Automatic trajectory planner for StereoElectroEncephaloGraphy procedures: a retrospective study. IEEE Trans Biomed Eng 60(4):968-995

12. Desikan RS, Ségonne F, Fischl B, Quinn BT, Dickerson BC, Blacker D, Buckner RL, Dale AM, Maguire RP, Hyman BT, Albert MS, Killiany RJ (2006) An automated labeling system for subdividing the human cerebral cortex on MRI scans into gyral based regions of interest. Neuroimage 31(3):968-980

13. Diehl B, Lüders HO (2000) Temporal lobe epilepsy: when are invasive recordings needed? Epilepsia 41(3):S61-S74

14. Essert C, Haegelen C, Lalys F, Abadie A, Jannin P (2012) Automatic computation of electrode trajectories for Deep Brain Stimulation: a hybrid symbolic and numerical approach. Int J Comput Assist Radiol Surg 7(4):517-532

15. Fleiss JL (1971) Measuring nominal scale agreement among many raters. Psychol Bull 76(5):378-382

16. Gering DT, Nabavi A, Kikinis R, Grimson WE, Hata N, Everett P, Jolesz FA, Wells WM (1999) An integrated visualization system for surgical planning and guidance using image fusion and interventional imaging. In: Taylor C, Colchester A (eds) International conference on medical image computing and computer assisted intervention (MICCAI), LNCS 1679. Springer, Berlin, pp 809-819

17. Hoffmann D, Lo Russo G (2008) Stereoelectroencephalography. In: Lüders HO (ed) Textbook of epilepsy surgery. Informa Healthcare, London, pp 945-959

18. Jenkinson M, Bannister PR, Brady JM, Smith SM (2002) Improved optimisation for the robust and accurate linear registration and motion correction of brain images. NeuroImage 17(2):825-841

19. Kahane P, Landrè E, Minotti L, Francione S, Ryvlin P (2006) The Bancaud and Talairach view on the epileptogenic zone: a working hypothesis. Epileptic Disord 8(2):S16-S26

20. Kilpatrick C, Cook M, Kaye A, Murphy M, Matkovic Z (1997) Non-invasive investigations successfully select patients for temporal lobe surgery. J Neurol Neurosurg Psychiatry 63(3):327-333

21. Kimmel R, Sethian JA (1998) Computing geodesic paths on manifolds. Proc Natl Acad Sci 95:8341-8435

22. Liu Y, Dawant BM, Pallavaram S, Neimat J S, Konrad PE, D'Haese PF, Datteri R, Landman BA, Noble JH (2012) A surgeon specific automatic path planning algorithm for deep brain stimulation. In: Holmes DRIII, Wong KH (eds) Medical imaging 2012: image-guided procedures, robotic interventions, and modeling, SPIE 83161D

23. Munari C (1987) Depth electrode implantation at Hôpital Sainte Anne, Paris. In: Engel J Jr (ed) Surgical treatment of the epilepsies. Raven Press Ltd, New York, pp 583-588

24. Munari C, Hoffmann D, Francione S, Kathane P, Tassi L, Lo Russo G, Benabid AL (1994) Stereo-electroencephalography methodology: advantages and limits. Acta Neurol Scand Suppl 152:56-67

25. Roche A, Malandain G, Pennec X, Ayache N (1998) The correlation ratio as a new similarity measure for multimodal image registration. In: Wells W, Colchester A, Delp S (eds) International conference 
on medical image computing and computer assisted intervention (MICCAI), LNCS1496. Springer, Berlin, pp 1115-1124

26. Seitel A, Engel M, Sommer CM, Radeleff BA, Essert-Villard C, Baegert C, Fangerau M, Fritzsche KH, Yung K, Meinzer HP, MaierHein L (2011) Computer-assisted trajectory planning for percutaneous needle insertions. Med Phys 38(6):3246-3259

27. Shamir RR, Joskowicz L, Tamir I, Dabool E, Pertman L, Ben-Ami A, Shoshan Y (2012) Reduced risk trajectory planning in imageguided keyhole neurosurgery. Med Phys 39(5):2885-2895

28. Shamir RR, Tamir I, Dabool E, Joskowicz L, Shoshan Y (2010) A method for planning safe trajectories in image-guided keyhole neurosurgery. In: Jiang T, Navab N, Pluim JPW, Viergever MA (eds) International conference on medical image computing and computer assisted intervention (MICCAI), LNCS 6363. Springer, Berlin, pp 457-464

29. Shi Y, Sun B, Lai R, Dinov I, Toga AW (2010) Automated sulci identification via intrinsic modeling of cortical anatomy. In: Jiang T, Navab N, Pluim JPW, Viergever MA (eds) International conference on medical image computing and computer assisted intervention (MICCAI), LNCS 6363. Springer, Berlin, pp 49-56

30. Smith SM, Jenkinson M, Woolrich MW, Beckmann CF, Behrens TE, Johansen-Berg H, Bannister PR, De Luca M, Drobnjak I, Flitney DE, Niazy RK, Saunders J, Vickers J, Zhang Y, De Stefano N, Brady JM, Matthews PM (2004) Advances in functional and structural MR image analysis and implementation as FSL. Neuroimage 23(1):S208-S219
31. Spencer SS, Sperling MR, Shewmon AD (1997) Intracranial electrodes. In: Engel J Jr, Pedley TA (eds) Epilepsy: a comprehensive textbook. Lippincott- Raven, Philadelphia, pp 1719-1747

32. Thadani VM, Williamson PD, Berger R, Spencer SS, Spencer DD, Novelly RA, Sass KJ, Kim JH, Mattson RH (1995) Successful epilepsy surgery without intracranial EEG recording: criteria for patient selection. Epilepsia 36(1):7-15

33. Viola P, Wells WM (1997) Alignment by maximization of mutual information. Int J Comp Vis 24(2):137-154

34. Vitter JS (1984) Faster methods for random sampling. Commun ACM 27(7):703-718

35. Yuan J, Chen Y, Hirsch E (2012) Intracranial electrodes in the presurgical evaluation of epilepsy. Neurol Sci 33:723-729

36. Zumsteg D, Wiese HG (2000) Presurgical evaluation: current role of invasive EEG. Epilepsia 41(3):S55-S60 